\title{
SEDOPTICA Newsletter
}

\section{Noticias de SEDOPTICA}

A continuación, recogemos las principales noticias y actividades de SEDOPTICA y de sus socios en el periodo de octubre a diciembre de 2017, así como las perspectivas para el próximo año.

\section{50 aniversario de SEDOPTICA}

SEDOPTICA cumple 50 años en 2018. Se trata de un gran motivo de celebración para la sociedad. Para comenzar esta celebración, os anunciamos la realización de un logo conmemorativo, diseñado por José Juan Castro, Presidente del Comité de Ciencias de la Visión, así como la publicación digital en la web de SEDOPTICA del primer número de la revista Óptica Pura y Aplicada - OPA, que puede descargarse desde la página web de SEDOPTICA en el siguiente enlace. Estamos trabajando para poder disponer de la colección completa de OPA de forma digital a lo largo del próximo año.
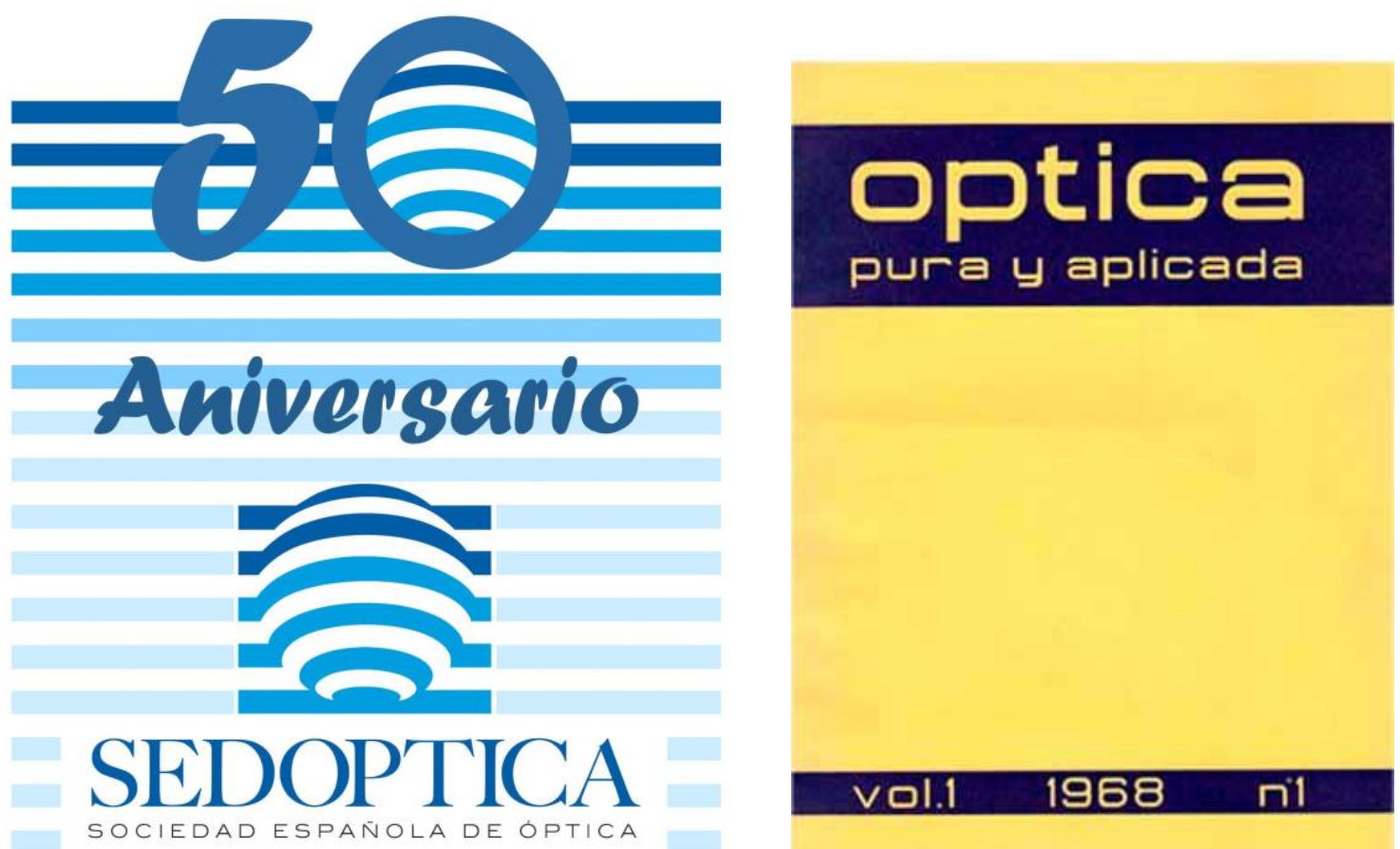

Fig. 1: Izquierda: Logo del 50o aniversario de SEDOPTICA. Derecha: portada del primer número de OPA en 1968, que puede descargarse desde el siguiente enlace. 


\section{Socios de SEDOPTICA}

El pasado día 30 de octubre de 2017 tuvo lugar en la Lonja de los Mercaderes de Valencia el acto de entrega de la 29a edición de los "Premios Rey Jaime I". En dicho acto Susana Marcos recibió el Premio Jaime I de Nuevas Tecnologías 2017 de manos de su Majestad la Reina. Nuestra más entusiasta enhorabuena a Susana y a todo su equipo por este importantísimo galardón. Este premio se une al reciente galardón de la Fundación Madrid+d a Carlos Dorronsoro, José Ramón Alonso y Susana Marcos, a la mejor patente por su invención "'Instrumento miniaturizado simulador de visión simultánea".

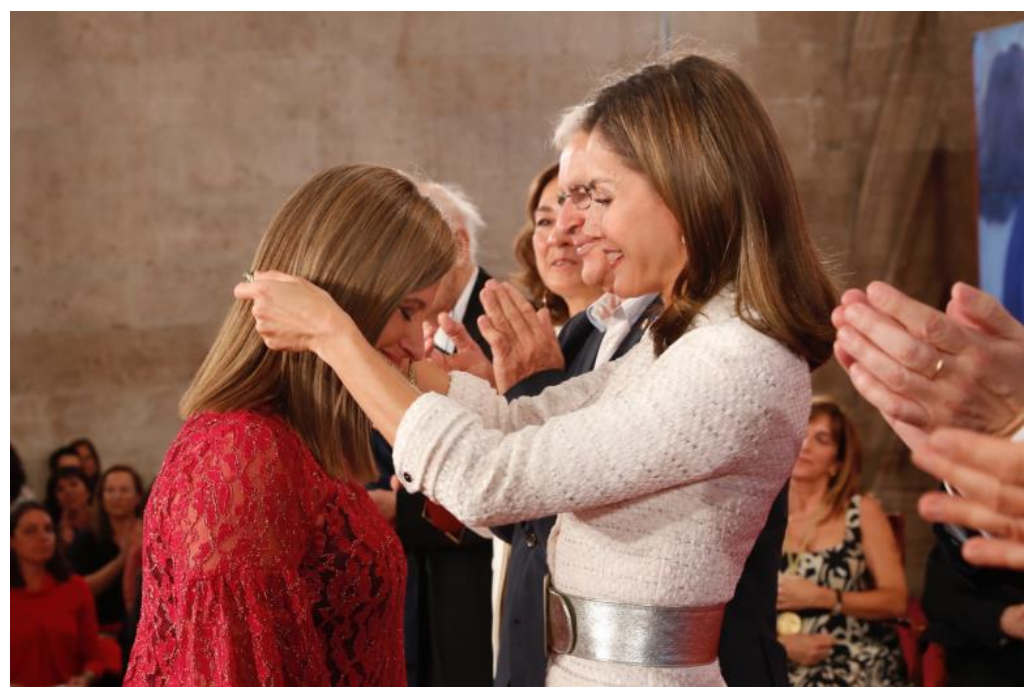

Fig. 2. Su Majestad la Reina impone la medalla del Premio Rey Jaime I de Nuevas Tecnologías 2017 a Susana Marcos. Fuente: http://www.casareal.es/ES/Actividades/Paginas/actividades actividades detalle.aspx?data=13351

Por otro lado, la revista Nature ha seleccionado a Lluis Torner como uno de los cuatro científicos españoles galardonados con el '2017 Nature Mentoring Awards'. Estos galardones, instaurados en 2005, se otorgan por regiones o países, y en 2017 se han otorgado por primera vez en España. Lluis Torner recibió el galardón en un acto celebrado en la Real Academia de Ciencias de Madrid, junto con los investigadores Margarita Salas, Carlos Belmonte y Carlos López-Otín. El acto, presentado por Sir Philip Campbell, Editorin-Chief de la revista Nature, y contó con la presencia e intervención de Dña. Carmen Vela, Secretaria de Estado de Investigación, Desarrollo e Investigación. Nuestra enhorabuena a Lluis.

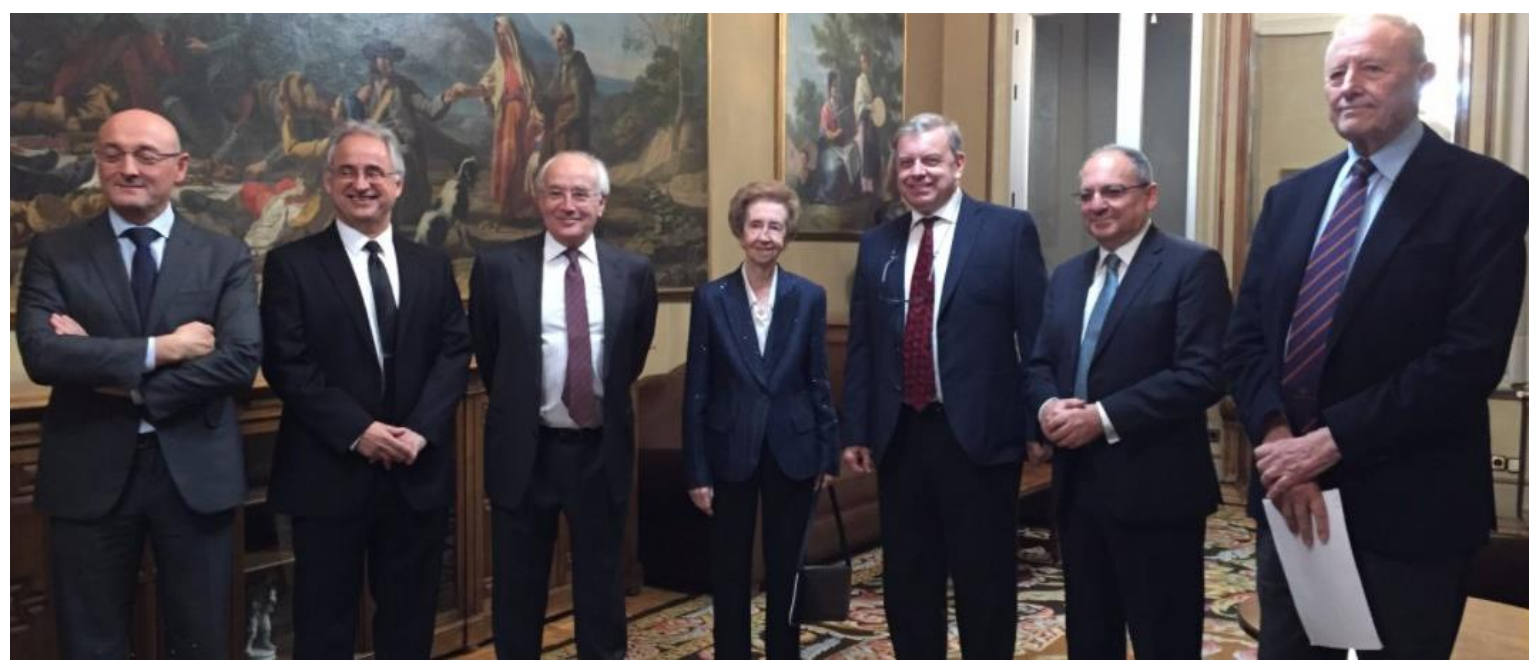

Fig. 3. Lluis Torner, junto a los otros premiados, y Sir Phillip Campbell, en el acto de entrega de los '2017 Nature Mentoring Awards'. Fuente: Nature: https://twitter.com/nature/status/939197407937597440?lang=es 
Finalmente, el pasado día 12 de diciembre, Juan Ignacio Cirac impartió la conferencia titulada "Ciencia y tecnologías Cuánticas de la Información" dentro del ciclo de conferencias Colloquium Marie Sklodowska Curie, en acto celebrado en el Instituto de Química Física Rocasolano, del Consejo Superior de Investigaciones Científicas (IQFR-CSIC). Al finalizar el acto, se hizo entrega al Prof. Cirac de la placa y diploma como Socio de Honor de SEDOPTICA.

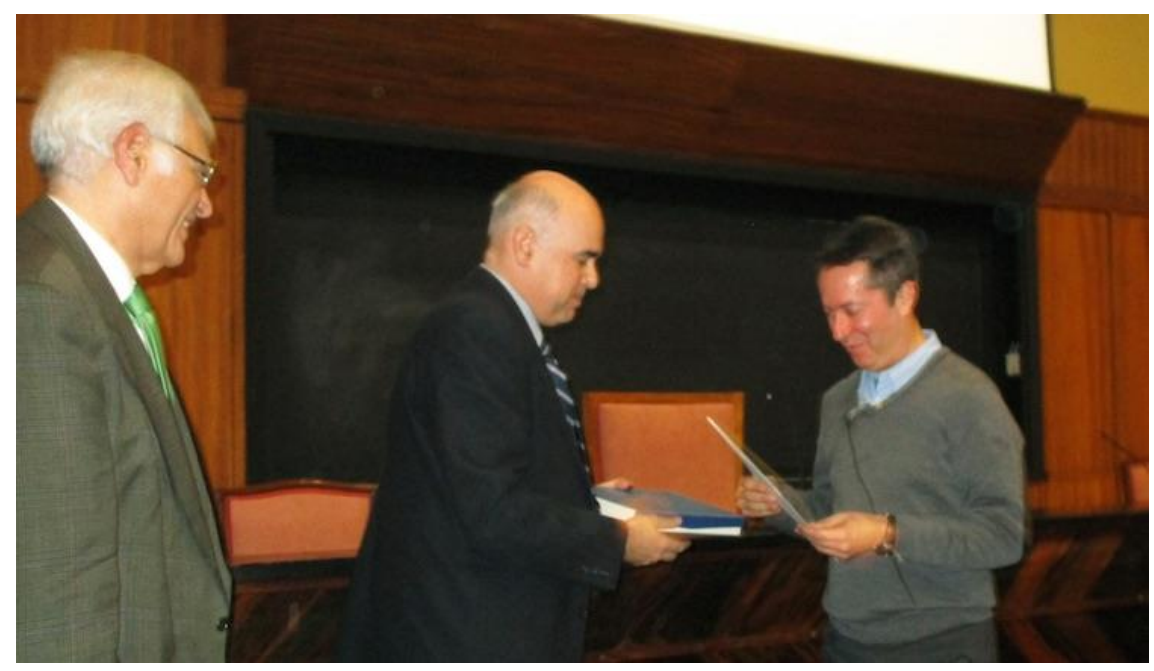

Fig. 4. Ignacio Moreno y Joaquín Campos (Presidente de SEDOPTICA en 2011-2014) hacen entrega de la placa y diploma como Socio de Honor de SEDOPTICA a Juan Ignacio Cirac, en el acto celebrado en el Instituto de QuímicaFísica Rocasolano (CSIC) el día 12 de diciembre de 2017.

\section{Cursos, Escuelas y Congresos}

El pasado día 15 de noviembre, tuvo lugar el Webminar Instrumentación avanzada en oftalmología y optometría, organizado por SECPhO en colaboración con el Comité de Ciencias de la Visión, y cuyos conferenciantes fueron Jaume Pujol, Pablo Artal y Susana Marcos.

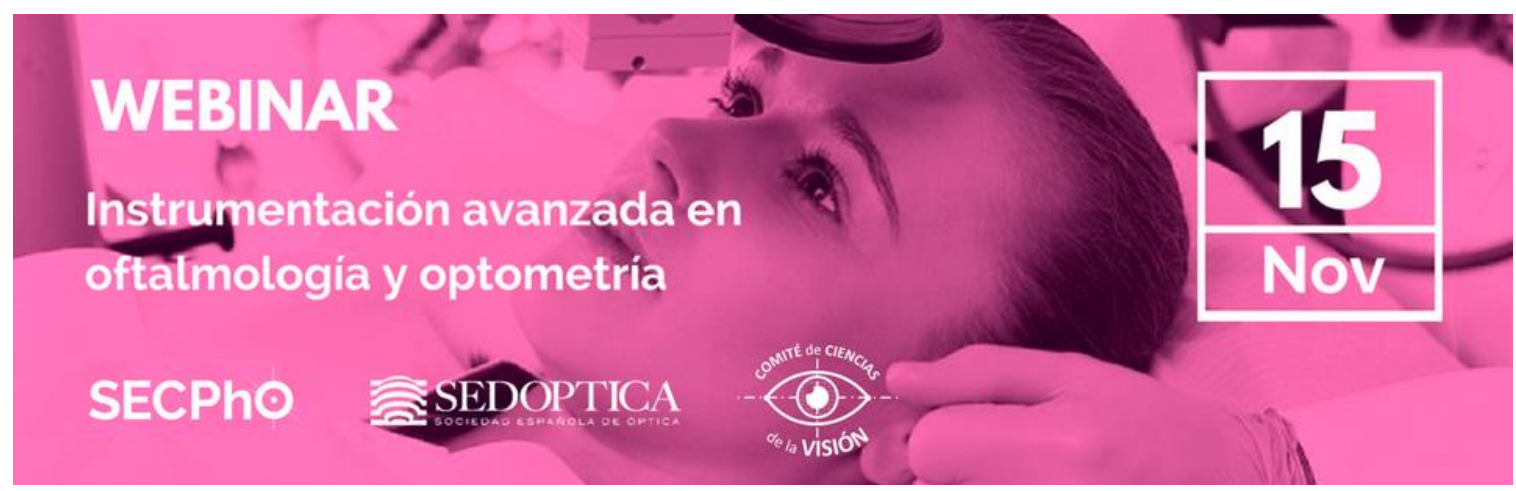

Fig. 5. Anuncio del Webminar de Instrumentación avanzada en oftalmología, y optometría.

En Bucaramanga (Colombia), se celebró el XV Encuentro Nacional de Óptica y VI Conferencia Andina y del Caribe en Óptica y sus Aplicaciones. El congreso, organizado por el Grupo de Óptica y Tratamiento de Señales coordinado por el profesor Jaime Meneses, tuvo su sede en la Universidad Industrial de Santander y contó con la presentación de 127 comunicaciones - 9 de ellas conferencias plenarias- y una duración de cinco días (del 20 al 24 de noviembre). La vicepresidenta de SEDOPTICA, María Sagrario Millán, participó en el congreso y asistió a la reunión convocada por Efraín Solarte, actual presidente de SOFIA (Sociedades de Óptica y Fotónica de Iberoamérica) sobre los avances en la elaboración de los estatutos y la evolución a partir de la anterior RIAO (Red Iberoamericana de Óptica). Entre otros temas, en el encuentro se hizo una valoración muy positiva de la revista Óptica Pura y Aplicada, de la que se destacó su creciente nivel de reconocimiento científico, su carácter de libre acceso por internet y un coste de publicación muy asequible, además de ofrecer la posibilidad de publicar en lengua española. 


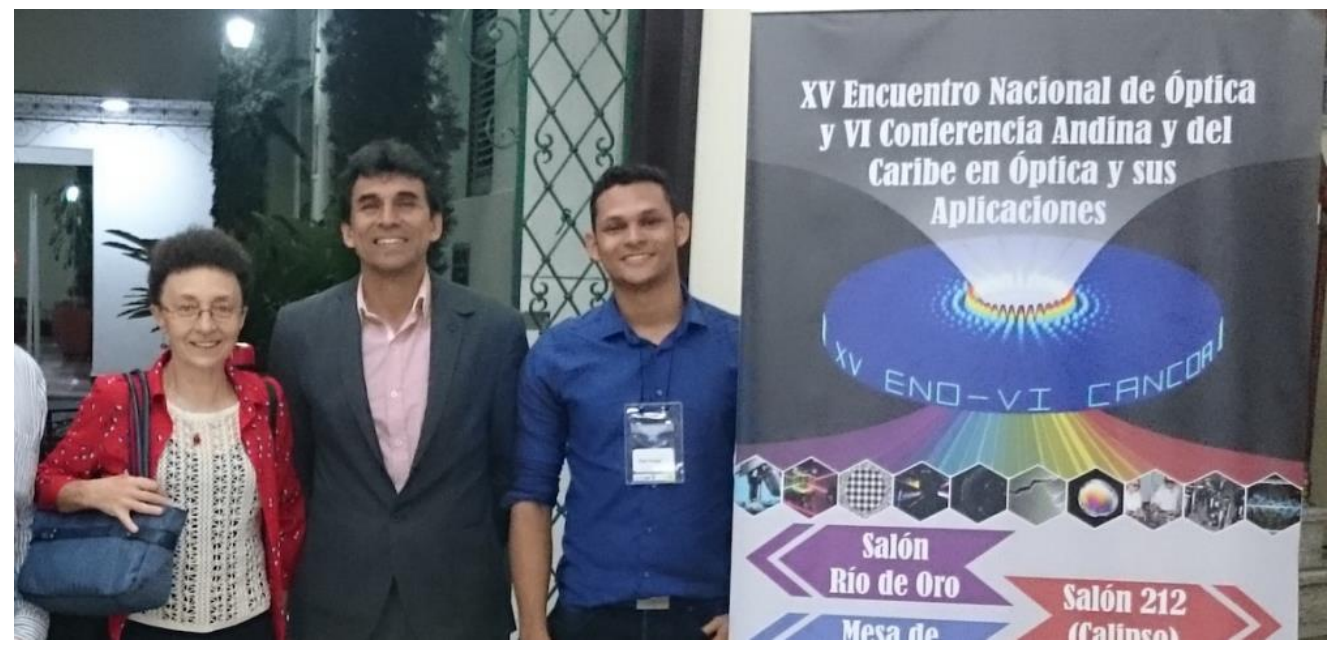

Fig. 6. María Sagrario Millán, Vicepresidenta de SEDOPTICA, junto a Jaime Meneses, organizador del congreso ENOCANCOA 2017 y un participante.

El año 2018 se inicia con una intensa actividad en la realización de jornadas y congresos. El próximo trimestre. La empresa VLC Photonics, empresa colaboradora de SEDOPTICA, organiza del 17 al 19 de enero el II Curso de Diseño de PICs - Photonic Integrated Circuits. El Comité de Espectroscopía organiza los días 15 y 16 de febrero en la Universidad de Málaga, las Jornadas de Ciencia y Tecnología ES-LIBS. Los días del 4 al 7 de marzo se realizará en Murcia el XI Workshop on Adaptive Optics for Industry and Medicine, organizado por Pablo Artal y su equipo. Y en el mes de mayo, se organiza en la Valencia la 20th European Conference on Integrated Optics - ECIO 2018, organizado por Pascual Muñoz y Pablo Sanchís.

El 16 de mayo de 2018 será el primer Día Internacional de la Luz. Desde SEDOPTICA hemos impulsado la creación de un Comité relacionado, a semejanza del que ya se organizó en el Año Internacional de la Luz, que coordine los esfuerzos y actividades que se vayan a hacer. Está ya prevista la celebración de un acto central en la Facultad de Física de la Universidad Complutense de Madrid.

$Y$ en el horizonte del próximo verano tenemos dos congresos de SEDOPTICA: la XII Reunión Nacional de Óptica - RNO, en Castellón de la Plana del 3 al 6 de julio, donde realizaremos una celebración especial del 50o aniversario de SEDOPTICA, y la XXVI Reunión Nacional de Espectroscopía (RNE) y X Congreso Ibérico de Espectroscopía, en Lisboa del 9 al 11 de julio de 2018.

Finalmente, deseamos recordar que el Comité de Técnicas de la Imagen ha convocado la VI edición del Premio Justiniano Casas de investigación en imagen óptica, en esta ocasión patrocinado por la empresa LASING S.A., empresa colaborada de SEDOPTICA. El plazo de presentación de los aspirantes al premio finaliza el día 20 de marzo de 2018, por lo que animamos a presentarse todos los que cumplan los requisitos.

Elche, diciembre de 2017

\section{Premio Justiniano Casas}

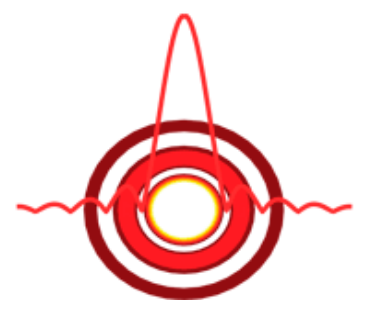

Fig. 7. Anuncio del Premio Justiniano Casas de investigación en imagen óptica, cuyas bases se encuentran en el siguiente enlace.

Ignacio Moreno

Presidente de SEDOPTICA

María S. Millán

Vicepresidenta de SEDOPTICA

Santiago Vallmitjana

Presidente anterior de SEDOPTICA

DOI: http://dx.doi.org/10.7149/OPA.50.4.i 